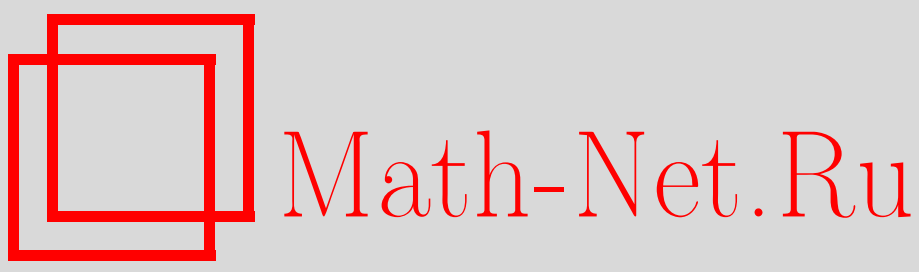

А. Н. Тюрин, Модели Дельцана пространств модулей, Изв. РАН. Сер. матем., 2003, том 67, выпуск 2, 167-180

DOI: https://doi.org/10.4213/im430

Использование Общероссийского математического портала Math-Net.Ru подразумевает, что вы прочитали и согласны с пользовательским соглашением

http://www . mathnet.ru/rus/agreement

Параметры загрузки:

IP : 34.229 .45 .116

26 апреля 2023 г., 06:37:59 
УДК 512.723

$$
\text { А. Н. Тюрин }
$$

\title{
Модели Дельцана пространств модулей
}

\begin{abstract}
Для каждого значения рода $g$ строятся гладкое полное рациональное поляризованное алгебраическое многообразие $\left(D M_{g}, H\right)$ и эффективный дивизор на нем с нормальными пересечениями компонент $D=\bigcup D_{i}$ такие, что для каждого пространства модулей $M_{\Sigma}(2,0)$ полустабильных топологически тривиальных векторных расслоений ранга 2 на алгебраической кривой $\Sigma$ рода $g$ существует голоморфный изоморфизм $f: M_{\Sigma}(2,0) \backslash K_{g} \rightarrow D M_{g} \backslash D$, где $K_{g}$ - куммерово многообразие якобиана кривой $\Sigma$, переводящий поляризацию многообразия $D M_{g}$ в тэта-дивизор многообразия модулей. Этот изоморфизм индуцирует изоморфизм пространств $H^{0}\left(M_{\Sigma}(2,0), \Theta^{k}\right)=H^{0}\left(D M_{g}, H^{k}\right)$.
\end{abstract}

Библиограб̆ия: 12 наименований.

\section{§1. Введение}

На последней конференции $\mathrm{GAEL}^{1}$ Б. Оксбери поставил вопрос о “топологической" идентификации многообразия модулей $M_{\Sigma}(2,0)$ с трехмерным комплексным проективным пространством $\mathbb{C P}^{3}$ для любой кривой $\Sigma$ рода 2 . Чтобы понять сушность этой проблемы, напомним, что как вешественное многообразие это пространство модулей является пространством $\operatorname{ClRep}\left(\pi_{1}(\Sigma)\right)$ классов представлений фундаментальной группы кривой $\pi_{1}(\Sigma)$ в $\mathrm{SU}(2)$. Проблема заключается в идентификации этого пространства с пространством $\mathbb{C P}^{3}$.

Известно, что комплексная структура на компактной римановой поверхности $\Sigma$ рода 2 индуцирует комплексную структуру на $\operatorname{ClRep}\left(\pi_{1}(\Sigma)\right)$ как на пространстве модулей $M_{\Sigma}(2,0)$ полустабильных ранга 2 голоморфных векторных расслоений с тривиальным детерминантом. В этой комплексной структуре пространство $\operatorname{ClRep}\left(\pi_{1}(\Sigma)\right)$ отождествляется с пространством $\mathbb{C P}^{3}$. Однако хотелось бы увидеть прямое отождествление пространства классов представлений $\operatorname{ClRep}\left(\pi_{1}(\Sigma)\right)$ с трехмерным проективным пространством.

В частности, для этого случая характерно, что:

1) многообразие модулей $M_{\Sigma}(2,0)$ как алгебраическое многообразие не зависит от выбора комплексной структуры на $\Sigma$;

2) многообразие модулей $M_{\Sigma}(2,0)$ рационально;

3) пространство конформных блоков (т. е. голоморфных сечений поляризации) не зависит от модулей кривой.

\footnotetext{
${ }^{1}$ Géométrie algébrique en liberté (9th edition, 19th-23rd March 2001), школа организована EAGER, EU project Contract No. HPRN-CT-2000-00099.
} 
Пространство ClRep $\left(\pi_{1}(\Sigma)\right)$ классов представлений фундаментальной групшы $\pi_{1}(\Sigma)$ в $\mathrm{SU}(2)$ допускает каноническую симплектическую форму $\Omega$, которая определяется чисто топологически (см. [2]). Следовательно, в этой ситуации естественно привлечь технику симплектической геометрии, а точнее, технику гамильтоновых действий торов, т. е. торической симплектической геометрии (см. монографию [8] - основной источник для технических деталей).

Напомним основные конструкции теории торических многообразий. Пусть $(M, \omega)$ - симплектическое многообразие размерности $2 n$ с гладким гамильтоновым действием $n$-мерного тора $T^{n}$, т. е. существует отображение $f: T^{n} \rightarrow \operatorname{Diff} M$ такое, что действия образов сохраняют форму $\omega$. Тогда координаты “действие угол" определяют отображение моментов

$$
\pi: M \rightarrow \Delta \subset \mathbb{R}^{n}
$$

образ которого есть выпуклый полиэдр $\Delta$ в евклидовом $n$-мерном пространстве. Этот полиэдр несет полную информацию о симплектической геометрии многообразия $(M, \omega)$, т.е. полиэдр $\Delta$ определяет само многообразие, симплектическую форму и $T^{n}$-действие (см. [1]).

Более того, если на многообразии $(M, \omega)$ задана предквантизация (см. [8]) и $M$ имеет структуру Ходжа, келеровой формой которой является $\omega$, то эта ходжева структура также может быть реконструирована.

Пространство $\operatorname{ClRep}\left(\pi_{1}(\Sigma)\right)$ также допускает хорошо известное гамильтоново действие тора $T^{3 g-3}$ (см., например, [7]). Разница в свойствах этого действия и описанного вьше стандартного гамильтонова действия тора кажется, на первьй взгляд, ничтожной, а именно заключается в следующем:

1) при $g>2$ пространство классов представлений $\operatorname{ClRep}\left(\pi_{1}(\Sigma)\right)$ особо, множеством особых точек является куммерово многообразие

$$
\text { Sing ClRep }\left(\pi_{1}(\Sigma)\right)=K_{g}=R_{g}^{\mathrm{U}(1)} / \pm \mathrm{id}
$$

т. е. пространство U(1)-представлений фундаментальной группы $\pi_{1}(C)$ с точностью до $\pm \mathrm{id} ;$

$2)$ действие $(3 g-3)$-тора гладко только над внутренними точками полиэдра $\Delta$, хотя и непрерывно всюду.

Например, при $g=2$ пространство $\operatorname{ClRep}\left(\pi_{1}(\Sigma)\right)$ допускает действие $T^{3}$, отображение моментов которого имеет в качестве образа $\Delta$ стандартный тетраэдр

$$
0 \leqslant t_{i} \leqslant 1, \quad i=1,2, \quad\left|t_{1}-t_{2}\right| \leqslant t_{3} \leqslant \min \left(t_{1}+t_{2}, 2-t_{1}-t_{2}\right)
$$

в стандартном евклидовом пространстве $\mathbb{R}^{3}$ с координатами $t_{1}, t_{2}, t_{3}$. Это частньй случай дельцановского полиэдра (см. [8]), который однозначно определяет дельцановское ходжево многообразие $D M_{2}$. В этом случае оно совпадает с $\mathbb{C P}^{3}$, а выделенным дивизором $\bigcup \mathbb{C P}_{i}^{2}$ для $i=0,1,2,3$ является объединение четырех 
плоскостей в общем положении. Назовем это проективное пространство моделью Дельцана пространства $\operatorname{ClRep}\left(\pi_{1}(\Sigma)\right)$.

Используя комплексную структуру на $\operatorname{ClRep}\left(\pi_{1}(\Sigma)\right)$, задаваемую комплексной структурой на $\Sigma$, и эквивариантную версию теоремы Дарбу-Вайнстейна, получим голоморфное отображение

$$
f: D M_{2} \backslash \bigcup_{i=0}^{4} \mathbb{C P}_{i}^{2} \rightarrow M_{\Sigma}(2,0) \backslash K_{2}
$$

Несмотря на то, что многообразия $D M_{2}$ и $M_{\Sigma}(2,0)$ как рациональные алгебраические многоообразия изоморфны $\mathbb{P}^{3}$, биголоморфное отображение $f(1.2)$ не может быть продолжено до голоморфного отождествления $\mathbb{C P}^{3}=D M_{2}=M_{\Sigma}(2,0)$, т. е. мы оказываемся в условиях ситуации бирациональной (симплектической) геометрии.

Цель настоящей работы - построить модель Дельцана для кривой произвольного рода с набором свойств, перечисленных в аннотации. Случай кривых рода 2 указывает нам для этого путь. Кроме того, рассматриваемая конструкция дает конечную цепочку элементарных "бирациональных" преобразований (флипов), переводяших модель Дельцана $D M_{g}$ в рациональное многообразие $\left(\mathbb{C P}^{3}\right)^{g-1}$, в точности такую же, как та, что возникает для торических многообразий в алгебраической геометрии при перестройках полиэдров (см. [5]).

Идея построить модель, родственную дельцановской, восходит к Дональдсону, в работе [3] которого родственное к $D M_{g}$ многообразие построено для нечетного (гладкого) случая $M_{\Sigma}(2,1)$ как имитация многообразия модулей для объяснения появления чисел Бернулли в формулах Верлинде.

В четном (негладком) случае наша конструкция близка к конструкции Джеффри и Уртюбиз из работы [12]. На самом деле, почти очевидно, что многообразие $D M_{g}$ совпадает с многообразием $P^{D}$ уртюбиза и Джеффри, но прямое доказательство этого факта пока не опубликовано.

\section{$\S 2$. Торическая структура на $\operatorname{ClRep}\left(\pi_{1}(\Sigma)\right)$}

Пусть $\Sigma$ - гладкая риманова поверхность рода $g$ с фундаментальной группой $\pi_{1}(\Sigma)$ и $C$ - простая замкнутая кривая на $\Sigma$. Гомотопический класс такой кривой определяет так называемую функиию Голдмана на $\operatorname{ClRep}\left(\pi_{1}(\Sigma)\right)$ :

$$
c_{C}: \operatorname{ClRep}\left(\pi_{1}(\Sigma)\right) \rightarrow[0,1] \subset \mathbb{R},
$$

сопоставляюшую представлению $\rho \in \operatorname{ClRep}\left(\pi_{1}(\Sigma)\right)$ число

$$
\frac{1}{\pi} \cos ^{-1}\left(\frac{1}{2} \operatorname{Tr}(\rho([C]))\right) \in[0,1]
$$


где $[C]$ - гомотопический класс кривой $C$. В. Голдман [2] показал, что функция $c_{C}$ - гамильтониан $U(1)$-действия на $\operatorname{ClRep}\left(\pi_{1}(\Sigma)\right)$ относительно симплектической структуры $\Omega$. (Точная формула для этого действия в геометрических терминах приведена, например, в [3].) Более того, если $C_{1}$ и $C_{2}$ - две непересекающиеся кривые, то

$$
\left\{c_{C_{1}}, c_{C_{2}}\right\}=0
$$

где скобки Пуассона определяются симплектической структурой $\Omega$; в частности, если $\left[C_{1}\right] \neq\left[C_{2}\right]$, то мы получаем гамильтоново действие двумерного тора $T^{2}=\mathrm{U}(1) \times \mathrm{U}(1)$ на пространстве $\operatorname{ClRep}\left(\pi_{1}(\Sigma)\right)$. Добавляя третью кривую, получаем действие трехмерного тора и т. д. Конечно, этот процесс остановится, так как хорошо известно, что каждое максимальное множество непересекающихся и гомотопически неэквивалентных окружностей содержит $3 g-3$ кривые. Фиксируем любое из таких множеств:

$$
\left\{C_{1}, \ldots, C_{3 g-3}\right\}
$$

Класс изотопии такого множества окружностей называется разметкой римановой поверхности. Легко видеть, что дополнение такого множества - это объединение

$$
\Sigma_{g} \backslash\left\{C_{1}, \ldots, C_{3 g-3}\right\}=\coprod_{i=1}^{2 g-2} P_{i}
$$

$2 g-2$ тринионов $P_{i}$, а каждый тринион является 2-сферой с тремя удаленными непересекающимися дисками:

$$
P_{i}=S^{2} \backslash\left(D_{1} \cup D_{2} \cup D_{3}\right)
$$

Такое представление римановой поверхности называется тринионным разложением.

Таким образом, любое тринионное разложение поверхности $\Sigma$ задается выбором максимального множества непересекающихся, нестягиваемых, попарно негомотопных гладких окружностей на $\Sigma$, содержащего $3 g-3$ таких окружностей $C_{1}, \ldots, C_{3 g-3} \subset \Sigma_{g}$, дополнение к которым состоит из $2 g-2$ тринионов $P_{j}$. Тип разложения задан его дуальным момивалентным графом $\Gamma\left(\left\{C_{i}\right\}\right)$, каждая вершина которого ассоциирована с тринионом $P_{i}$, а ребро, соединяющее $P_{i}$ и $P_{j}$, ассоциировано с их общей гранищей $C_{l}$ - окружностью такой, что $C_{l} \subset \partial P_{i} \cap \partial P_{j}$. Таким образом, изотопический класс тринионного разложения однозначно задан тривалентным графом Г рода $g$.

С другой стороны, любой тривалентный граф $\Gamma$ с множеством вершин $V(\Gamma)$ и множеством ребер $E(\Gamma)$ определяет тело с ручками $\widetilde{\Gamma}$, т. е. 3 -многообразие с гранищей $\partial \widetilde{\Gamma}=\Sigma_{\Gamma}$ (римановой поверхностью рода $g$ с тринионным разложением), при помоши "раздутия" (см. [4]): раздуваем ребра графа Г до трубок и вершины - до маленьких 2-сфер. Получим риманову поверхность $\Sigma_{\Gamma}$ рода $g$ с трубкой $\widetilde{e}$ вместо 
каждого ребра $e \in E(\Gamma)$ и тринионом $\widetilde{v}$ вместо каждой вершины $v \in V(\Gamma)$. Изотопические классы меридианов трубок определяют $3 g-3$ непересекаюшихся, нестягиваемых, попарно неизотопных окружностей $\left\{C_{e}\right\}$ для $e \in E(\Gamma)$ и тринионное разложение поверхности $\Sigma$.

Таким образом, геометрически каждая риманова поверхность с разметкой $\left\{C_{i}\right\}$ эквивалентна тривалентному графу $\Gamma$; будем обозначать ее символом $\Sigma_{\Gamma}$. Для такой поверхности имеем отображение пространства классов представлений в евклидово пространство

$$
c_{\Gamma}: \operatorname{ClRep}\left(\pi_{1}(\Sigma)\right) \rightarrow \mathbb{R}^{3 g-3}=\mathbb{R}^{E(\Gamma)}
$$

с фиксированными координатами $\left(c_{1}, \ldots, c_{3 g-3}\right)$, пронумерованными элементами множества $E(\Gamma)$, задаваемое формулами $c_{i}=c_{C_{i}}$.

Тогда:

1) отображение $c_{\Gamma}$ - вешественная поляризация динамической системы с фазовым пространством $\left(\operatorname{ClRep}\left(\pi_{1}(\Sigma)\right), k \cdot \Omega\right)$;

2) $c_{i}$ - координаты действия гамильтоновой системы;

3) $c_{\Gamma}$ - отображение моментов для гамильтонова (но не всюду гладкого) действия тора $T^{3 g-3}$ на $\operatorname{ClRep}\left(\pi_{1}(\Sigma)\right)$ :

$$
\operatorname{ClRep}\left(\pi_{1}(\Sigma)\right) \times T^{3 g-3} \rightarrow \operatorname{ClRep}\left(\pi_{1}(\Sigma)\right)
$$

описанного в работе [7];

4) образ пространства $\operatorname{ClRep}\left(\pi_{1}(\Sigma)\right)$ относительно отображения $c_{\Gamma}$ - выпуклый полиэдр $\Delta_{\Gamma} \subset[0,1]^{3 g-3}$;

$5)$ симплектический объем пространства $\operatorname{ClRep}\left(\pi_{1}(\Sigma)\right)$ равен евклидову объему полиэдра $\Delta_{\Gamma}$ :

$$
\int_{\operatorname{ClRep}\left(\pi_{1}(\Sigma)\right)} \Omega^{3 g-3}=\operatorname{Vol} \Delta_{\Gamma}=\frac{2 \zeta(2 g-2)}{(2 \pi)^{g-1}} .
$$

Функции $c_{i}$ непрерывны на всем пространстве $\operatorname{ClRep}\left(\pi_{1}(\Sigma)\right)$ и гладки над $(0,1)$. Напомним, что такое гамильтоново действие тора на $\operatorname{ClRep}\left(\pi_{1}(\Sigma)\right)$ задано замкнутым тривалентным графом $\Gamma$ рода $g$ и приводит к следующему набору геометрических объектов:

1) выпуклый полиэдр $\Delta_{\Gamma} \subset[0,1]^{3 g-3}$;

2) часть границы $P_{r}=\partial \Delta_{\Gamma} \cap \partial[0,1]^{3 g-3} \subset \partial \Delta_{\Gamma}$;

3) часть границы выпуклого полиэдра $P_{K}=c_{\Gamma}\left(K_{g}\right) \subset \Delta_{\Gamma}$;

4) открытое подмножество $\Delta_{\Gamma}^{0}=\Delta_{\Gamma} \backslash\left(P_{r} \cup P_{K}\right) \subset[0,1]^{3 g-3}$;

5) открытое торическое пространство

$$
c_{\Gamma}^{-1}\left(\Delta_{\Gamma}^{0}\right)=\operatorname{ClRep}\left(\pi_{1}(\Sigma)\right)^{0} \subset \operatorname{ClRep}\left(\pi_{1}(\Sigma)\right),
$$

относительно компактное при отображении моментов $c_{\Gamma}: \operatorname{ClRep}\left(\pi_{1}(\Sigma)\right)^{0} \rightarrow \Delta_{\Gamma}^{0}$.

Опишем эти геометрические объекты в следуюшем параграфе. 


\section{§3. Комбинаторные конструкции}

Будем пользоваться понятиями и обозначениями, введенными в [6]. Напомним, что любой замкнутый тривалентный граф $\Gamma$ задается множеством вершин $V(\Gamma)$ и "квадратичной формой инщиденщии". Пусть $\mathbb{Z}^{V(\Gamma)}$ - свободный $\mathbb{Z}$-модуль всех формальных линейных комбинаций вершин с коэффициентами из $\mathbb{Z}$. (Конечно, множество вершин является выделенным базисом этого модуля.) Тогда матрица инциденции $q_{\Gamma}-$ симметрическая матрица с элементами $\alpha_{v_{i}, v_{j}}$, равными числу ребер между вершинами $v_{i}, v_{j} \in V(\Gamma)$.

Конечно, группа перестановок множества $V(\Gamma)$ действует на такие матрицы перестановками строк и столбцов.

Напомним (см. [6]), что графф Г называется гиперболическим, если существуют два подмножества $V_{+}, V_{-} \subset V(\Gamma)$ такие, что подпространства $\mathbb{Z}^{V_{ \pm}}$изотопны относительно формы $q_{\Gamma}$. Матрищы форм таких графов имеют блочный вид:

$$
q_{\Gamma}=\left(\begin{array}{cccc}
0 & 0 & * & * \\
0 & 0 & * & * \\
* & * & 0 & 0 \\
* & * & 0 & 0
\end{array}\right)
$$

где блоки

$$
\left(\begin{array}{cc}
* & * \\
* & *
\end{array}\right) \in \operatorname{Hom}_{\mathbb{Z}}\left(\mathbb{Z}^{V_{+}}, \mathbb{Z}^{V_{-}}\right)
$$

задают отождествление

$$
*: V_{+} \leftrightarrow V_{-}
$$

Множество ребер гиперболического графа $E(\Gamma)$ может быть представлено как объединение непересекаюшихся троек с обшими вершинами:

$$
E(\Gamma)=\bigcup_{v \in V_{+}} E(\Gamma)_{v}
$$

где $E(\Gamma)_{v}$ - три ребра из вершины $v \in V_{+}$.

Далее рассмотрим риманову поверхность $\Sigma_{\Gamma}$ как результат раздутия графа $E(\Gamma)$ и подмножество

$$
\Sigma_{+}=\bigcup_{v \in V_{+}} \tilde{v} \subset \Sigma_{\Gamma}=\bigcup_{v \in V_{+} \bigcup V_{-}=V(\Gamma)} \tilde{v}
$$

которое назовем половиной римановой поверхности $\Sigma_{\Gamma}$ (см. [6]).

Все эти конструкции применимы к любому тривалентному граффу, в том числе и к несвязному. В частности, рассмотрим несвязное объединение

$$
\Theta^{g-1}=\Theta \sqcup \cdots \sqcup \Theta .
$$

Этот тривалентный граф рода $g$ определяет риманову поверхность

$$
\Sigma_{\Theta^{g-1}}=\Sigma_{\Theta} \sqcup \cdots \sqcup \Sigma_{\Theta}
$$


- несвязное объединение $g-1$ копии римановой поверхности рода 2 со стандартным тринионным разложением, соответствуюшим графу $\Theta$.

Фиксируем один тринион в тринионном разложении каждой копии поверхности $\Sigma_{\Theta}$ и обозначим соответствующее множество вершин символом $V_{+} \subset V\left(\Theta^{g-1}\right)$, а его дополнение - символом $V_{-}$. На эти множества натягиваются изотропные относительно $q_{\Theta g-1}$ подмодули. Следовательно, граф $\Theta^{g-1}$ гиперболический с естественной идентификацией $*$, сопоставляющей триниону $\widetilde{v} \mathrm{c} v \in V_{+}$второй тринион компоненты $\Sigma_{\Theta}$.

Далее, половина римановой поверхности $\Theta^{g-1}$ есть объединение

$$
\Sigma_{+}=\bigcup_{v \in V_{+}} \widetilde{v} \subset \Sigma_{\Theta^{g-1}},
$$

которое в точности совпадает с половиной римановой поверхности $\Sigma_{\Gamma}$ :

$$
\Sigma_{\Gamma} \supset \Sigma_{+} \subset \Sigma_{\Theta^{g-1}} .
$$

\section{§4. Пространства классов представлений}

Пространства $\operatorname{ClRep}\left(\pi_{1}(\Sigma)\right)$ и $\left(\operatorname{ClRep}\left(\pi_{1}(\Sigma)\right)\right)^{g-1}-$ симплектические с торическими структурами, определяемыми графами $\Gamma$ и $\Theta^{g-1}$ (см. $\left.\S 1\right)$, с отображениями моментов

$$
\begin{aligned}
c_{\Gamma} & : \operatorname{ClRep}\left(\pi_{1}(\Sigma)\right) \rightarrow \Delta_{\Gamma}, \\
c_{\Theta^{g-1}} & :\left(\operatorname{ClRep}\left(\pi_{1}(\Sigma)\right)\right)^{g-1} \rightarrow \Delta_{\Theta^{g-1}} .
\end{aligned}
$$

ПРЕДЛОЖЕНИЕ 4.1. Полиәдр $\Delta_{\Theta g-1}$ есть прямое произведение $g-1$ копии тетраэдра $\Delta_{\Theta}$ :

$$
\Delta_{\Theta^{g-1}}=\prod_{v \in V_{+}} \Delta_{\Theta}
$$

Более того, пусть $\operatorname{ClRep}\left(\pi_{1}\left(\Sigma_{+}\right)\right)$- пространство классов $\mathrm{SU}(2)$-представлений фундаментальной группы половины римановой поверхности $\Sigma_{\Theta^{g-1}} ;$ это пространство допускает отображение

$$
c_{\partial \Sigma_{+}}: \operatorname{ClRep}\left(\pi_{1}\left(\Sigma_{+}\right)\right) \rightarrow \Delta_{\Theta^{g-1}} .
$$

ПРЕДЛОЖЕНИЕ 4.2. Отображсение $c_{\partial \Sigma_{+}}$является изоморфизмом.

ДокАЗАТЕЛЬСТво для случая $g=2$ содержится в доказательстве предложения 3.1 из [7]. Таким образом, утверждение проверяется для каждой компоненты несвязного графа, а тем самым, и для любого рода.

Вложение $\Sigma_{+} \hookrightarrow \Sigma_{\Gamma}$ индуцирует отображение ограничения

$$
r: \operatorname{ClRep}\left(\pi_{1}(\Sigma)\right) \rightarrow \operatorname{ClRep}\left(\pi_{1}\left(\Sigma_{+}\right)\right),
$$

а отображение $c_{\Gamma}$ раскладывается в композицию:

$$
c_{\Gamma}=r \circ c_{\partial \Sigma_{+}},
$$

так как граница половины $\partial \Sigma_{+}$совпадает с полным набором $\left\{C_{e}\right\}$ для $e \in E(\Gamma)$. Отсюда получаем 
ПРЕДЛОЖЕНИЕ 4.3. Полиэдр $\Delta_{\Gamma}$ содержится в образе отображения $c_{\partial \Sigma_{+}}$. Следовательно, $\Delta_{\Gamma} \subset\left(\Delta_{\Theta}\right)^{g-1}$.

Теперь уже легко проверить следующее хорошо известное утверждение (см., например, $[9$, предложение 33.5$])$.

ПРЕДЛОЖЕНИЕ 4.4. Для описания полиэдра $\Delta_{\Gamma}$ необходимо:

1) рассмотреть произведение всех тетраэдров, соответствующих тринионам;

2) наложить линейные условия, соответствующие равенствам склейки тринионов.

СлЕДСтвИЕ 4.5. 1) Шаг 1) предложения 4.4 для $\Delta_{\Gamma}$ не отличается от соответствующего шага для $\left(\Delta_{\Theta}\right)^{2}$.

2) При шаге 2) предложения 4.4 заменяем равенства соответствующими неравенствами.

Для описания модели Дельцана необходимо элементарными преобразованиями

перестроить полиэдр $\Delta_{\Gamma}$ в $\left(\Delta_{\Theta}\right)^{g-1}$. Комбинация этих преобразований соответствует индукции по роду.

\section{§5. Перестройки полиэдров}

Ограничимся специальным графом рода $g$, так называемым графом мультитэma $g \Theta$ (см. рис. 1-3 из работы [6]). Это - вертикальный овал $O$ с $g-1$ горизонтальной перемычкой

$$
\left\{e_{g-1}, e_{g}, \ldots, e_{2 g-3}\right\} .
$$

Этот граф симметричен относительно вертикальной оси $a_{0}$, и мы обозначим символом $i_{0}: g \Theta \rightarrow g \Theta$ отражение относительно этой оси. На левой стороне графа лежат пронумерованные сверху вниз вершины

$$
v_{1}, \ldots, v_{g-1}
$$

Выберем половину множества вершин следующим образом:

$$
V_{+}=\left\{v_{1}, i_{0}\left(v_{2}\right), v_{3}, i_{0}\left(v_{4}\right), \ldots\right\} \text {. }
$$

Дополнительную половину множества вершин зададим равенством $V_{-}=i_{0}\left(V_{+}\right)$. При таком делении вершин граф $g \Theta$ становится гиперболическим вида (3.1) с изотропными подпространствами $\mathbb{Z}^{V_{ \pm}}$и инволюцией отождествления $*=i_{0}(3.3)$. Форма графа выделяет подмножество ребер на левой стороне овала $O$ :

$$
\left\{e_{1}, e_{2}, \ldots, e_{g-2} \mid e_{i}=\partial\left(v_{i}\right) \cap \partial\left(i_{0}\left(v_{i+1}\right)\right)\right\}
$$

и показывает, что только эти ребра графа $\left\{e_{1}, e_{2}, \ldots, e_{g-2}\right\}$ участвуют в конструкции нетривиальных комбинаторных флипов. Каждое такое ребро $e_{i}$ определяет координату $t_{3}^{i}$ в $\mathbb{R}_{i}^{3}$ и координату $t_{3}^{i+1}$ в $\mathbb{R}_{i+1}^{3}$. 
Рассмотрим случай $g=3$.

В этом случае множество горизонтальных ребер (5.1) - это $\left\{e_{2}, e_{3}\right\}$, а множество вершин (5.2) есть $\left\{v_{1}, v_{2}\right\}$. Множество вершин (5.3) - это

$$
V_{+}=\left\{v_{1}, i_{0}\left(v_{2}\right), v_{3}, i_{0}\left(v_{4}\right)\right\}
$$

а множество (5.4) есть $\left\{e_{1}\right\}, e_{1}=\partial\left(v_{1}\right) \cap \partial\left(i_{0}\left(v_{2}\right)\right)$.

Теперь опишем ограничения 2 ) следствия 4.5. Рассмотрим следуюшие инволюции пространства $\mathbb{R}^{6}=\mathbb{R}_{1}^{3} \times \mathbb{R}_{2}^{3}$ :

1) перестановку трехмерных компонент: $i_{12}\left(\mathbb{R}_{1}^{3}\right)=\mathbb{R}_{2}^{3}$;

2 ) перестановку двух координат в каждом из трехмерных пространств $\mathbb{R}_{1}^{3}$ и $\mathbb{R}_{2}^{3}$ : $i_{e_{1}}\left(t_{3}^{1}\right)=\left(t_{3}^{2}\right)$.

Напомним, что ограничения 1) предложения 4.4 уже учтены:

$$
\left|t_{1}^{i}-t_{2}^{i}\right| \leqslant t_{3}^{i} \leqslant t_{1}^{i}+t_{1}^{i}, \quad i=1,2 .
$$

Теперь склеим тринионы $v_{1}$ и $v_{2}$ вдоль $e_{1}$. Легко видеть, что верно следующее предложение.

ПРЕДЛОЖЕНИЕ 5.1. Ограничения 2) следствия 4.5 әквивалентны условиЯM

$$
\left|t_{1}^{i}-t_{2}^{i}\right| \leqslant t_{3}^{j} \leqslant t_{1}^{i}+t_{1}^{i}, \quad i \neq j .
$$

Отсюда немедленно получаем следующую теорему.

ТеОРема 5.2. Полиәдр моментов задается условиями

$$
\Delta_{3 \Theta}=\left(\Delta_{\Theta}\right)^{2} \cap i_{e_{1}}\left(\left(\Delta_{\Theta}\right)^{2}\right) .
$$

Действительно, инволюция $i_{12}$ сохраняет полиэдр $\left(\Delta_{\Theta}\right)^{2}$. Следовательно, условие (5.6) является геометрической интерпретацией неравенств (5.5).

Напомним, что тетраэдр $\Delta_{\Theta}-$ это выпуклая линейная оболочка множества $S$, состоящего из четырех точек в $\mathbb{R}^{3}$ :

$$
\Delta_{\Theta}=\langle(0,0,0),(0,1,1),(1,0,1),(1,1,0)\rangle
$$

Следовательно, $\left(\Delta_{\Theta}\right)^{2}$ также является выпуклой оболочкойшестнадцати точек

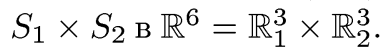

ПРЕДЛОЖЕНИЕ 5.3. Полиэдр $\Delta_{3 \Theta}$ есть выпуклая линейная оболочка точек

$$
\left\{\left(*, *^{\prime}, 0, *, *^{\prime}, 0\right)\right\} \cup\left\{\left(*, *^{\prime}, 1, *, *^{\prime}, 1\right)\right\} .
$$

Здесь символ $*$ означает свободный выбор из множеств $*=\{0,1\}$ и $*^{\prime}=\{0,1\}$. Действительно, неравенства $t_{3}^{1} \neq t_{3}^{2}$ индуцируют все эти запреты (5.5). 
Описание этой ситуации станет более понятным, если привлечь вещественную алгебраическую геометрию. А именно, пусть $C$ - вещественная алгебраическая кривая рода $g=2$ с вещественной тэта-характеристикой; ее куммерова поверхность является вещественной квартикой $K_{2}$ с шестнадшатью комплексно-сопряженными двойными особыми точками $\left\{p_{1}, \ldots, p_{16}\right\}$, называемыми нодами, в $\mathbb{C P}^{3}$. В аффинной части вокруг вещественной линейной оболочки этих нод-точек пространство $\mathbb{C P}^{3}$ представляется как $\mathbb{R}^{6}=\mathbb{R}^{3} \times i \mathbb{R}^{3}$. Тогда выпуклая оболочка

$$
\left\langle p_{1}, \ldots, p_{16}\right\rangle=\Delta_{\Theta^{2}}=\Delta_{\Theta} \times \Delta_{\Theta} \subset \mathbb{R}^{6}
$$

есть дельцановский полиэдр многообразия $\left(\mathbb{C P}^{3}\right)^{2}$ с естественным действием тора. Напомним, что через каждую вершину $p_{i}$ проходят шесть прямых с шестью вершинами на каждой, как и предписывает классическая конфигурация Куммера $16_{6}$. Из этого описания ясно видны восемь вершин выпуклого полиэдра $\Delta_{3 \Theta}$. Понятно, как сделать эти полиэдры целыми.

Проведем индукцию по $g$.

Дальнейшая стратегия теперь проста и естественна - строим последовательность полиэдров как последовательность аппроксимаций полиэдра $\Delta_{g \Theta}$ :

1) первую аппроксимацию $\left(\Delta_{\Theta}\right)^{g-1}$;

2) вторую аппроксимацию $\left(\Delta_{\Theta}\right)^{g-3} \times \Delta_{3 \Theta}$;

3) $i$-ю аппроксимацию $\left(\Delta_{\Theta}\right)^{g-i} \times \Delta_{i \Theta}$;

$4)$ и, наконец, последнюю $(g-1)$-ю аппроксимацию, совпадающую с самим полиэдром $\Delta_{g \Theta}$.

Так шаг за шагом мы продвигаемся к полиэдру с номером $g$. Заметим, что на предпоследнем шаге мы имеем:

1) полиэдр $\Delta_{\Theta} \times \Delta_{(g-2) \Theta} \subset \mathbb{R}^{3} \times \mathbb{R}^{3(g-2)}$, соответствующий несвязному объединению $\Theta \cup(g-2) \Theta$;

2 ) во второй компоненте $\Delta_{(g-2) \Theta}$ тринион $v_{l}$, выделенный на предыдушем шаге индукции как самый нижний тринион в $\Delta_{(g-3) \Theta}$, т. е. имеем разложение $\mathbb{R}^{3(g-2)}=$ $\mathbb{R}_{l}^{3} \times \mathbb{R}^{3(g-3)}$

3) выделенные ребра $e \in E(\Theta)$ и $e \in E_{v_{l}}((g-3) \Theta)$, вдоль которых склеиваем поверхности $\Sigma_{\Theta}$ и $\Sigma_{(g-1) \Theta}$;

4 ) выделенные координатные оси: $t_{3}$-ось в $\mathbb{R}^{3}$ и $t_{3}^{l}$-ось в $\mathbb{R}_{l}^{3}$, соответствующие ребру $e$, в разных трехмерных пространствах со стандартными координатами $\left(t_{1}\right.$, $\left.t_{2}, t_{3}\right)$ в $\mathbb{R}^{3}$ и $\left(t_{1}^{l}, t_{2}^{l}, t_{3}^{l}\right)$ в $\mathbb{R}_{l}^{3}$.

Теперь ограничения склейки в точности такие же, как в (5.5): в только что описанных обозначениях

$$
\left|t_{1}-t_{2}\right| \leqslant t_{3}^{l} \leqslant t_{1}+t_{2}, \quad\left|t_{1}^{l}-t_{2}^{l}\right| \leqslant t_{3} \leqslant t_{1}^{l}+t_{1}^{l}
$$

Используя те же аргументы, что при доказательстве предложения 5.1, получаем следующее предложение. 
ПРЕДЛОЖЕНИЕ 5.4. Полиэдр $\Delta_{g \Theta}-$ это выпуклая оболочка $2^{g}$ точек

$$
\left\{\left(*, *^{\prime}, 0, *, *^{\prime}, 0, *, \ldots, *\right)\right\} \cup\{(*, *, 1, *, *, 1, *, \ldots, *)\} \subset \mathbb{R}^{3(g-1)}
$$

c тем же описанием $*$, $*^{\prime}$ и остальных символов, что в формуле (5.7).

Другое описание полиэдра моментов как подполиэдра в $\Delta_{\Theta}^{g-1}$ дал Флорентино в работе [10].

Напомним (см., например, [8]), что выпуклый полиэдр $\Delta \subset \mathbb{R}^{n}$ дельцановский, если для каждой вершины $v$ сушествует целочисленная $(n \times n)$-матрица $A$ с детерминантом \pm 1 такая, что отображение

$$
t \in \mathbb{R}^{n} \rightarrow A t-v
$$

переводит окрестность вершины $v \in \Delta$ в окрестность нуля в $\mathbb{R}^{n}$.

Иначе говоря, полиэдр $\Delta \subset \mathbb{R}^{n}$ - дельцановский, если:

1) его 1-остов (объединение ребер) есть $n$-валентньй граф̆ $\Gamma$ (топологическое условие);

2) множество $E(\Gamma)_{v} \subset E(\Gamma)$ ребер, проходящих через вершину $v \in V(\Gamma)$, составляет рациональньй базис в $\mathbb{R}^{n}$.

Конечно, прямое произведение дельцановских полиэдров является дельцановским.

ПРЕДЛОЖЕНИЕ 5.5. Полиәдр $\Delta_{g \Theta} \subset \mathbb{R}^{3 g-3}-$ дельцановский.

Для доказательства предложения мы опять используем индукцию по роду $g$. На самом деле, и для шага индукции, и для базы достаточно рассмотреть случай $g=2$. Здесь мы имеем единичный куб $C=[0,1]^{3}$ с восемью вершинами. Чтобы построить из них тетраэдр $\Delta_{2}$, рассмотрим начало координат $(0,0,0)$ и выберем все вершины на расстоянии $\sqrt{2}$ от начала. Выпуклая линейная оболочка этих четырех вершин и есть тетраэдр $\Delta_{2}$. Та же конструкция применима для $\mathbb{R}^{6}=$ $\mathbb{R}^{3} \times \mathbb{R}^{3}$ : выбираем вершины куба на расстоянии 2 от начала и берем вьпуклую оболочку, и так далее. Наконец, для рода $g$ полиэдр $\Delta_{g \Theta} \subset \mathbb{R}^{3 g-3}$ есть выпуклая оболочка вершин единичного куба в $\mathbb{R}^{3 g-3}$, находяшихся на расстоянии $\sqrt{2 g-2}$ от начала координат. Так мы получаем все полиэдры.

\section{§6. Дельцановская модель}

Теперь мы имеем точное описание образа отображения моментов гамильтонова действия тора на $\operatorname{ClRep}\left(\pi_{1}(\Sigma)\right)$. Оказалось, что это - дельцановский полиэдр. Следовательно, согласно основной теореме теории Дельцана существует гладкое алгебраическое многообразие $D M_{g}$ с регулярным гамильтоновым действием на нем тора $T^{3 g-3}$.

ОПРЕдЕЛЕниЕ 6.1. Гладкое алгебраическое многообразие с ходжевой метрикой $D M_{g}$ назьвается моделью Дельцана пространства $\operatorname{ClRep}\left(\pi_{1}(\Sigma)\right)$ (или $\left.M_{C}(2,0)\right)$. 
Прямая конструкция этого рационального многообразия описана во многих работах, но книга [8] является лучшим источником этого описания.

Хорошо известен набор его характеристических свойств:

1) гладкое алгебраическое многообразие $D M_{g}$ имеет каноническую поляризацию $H$;

2) размерность пространства сечений $H^{0}\left(D M_{g}, H^{k}\right)$ может быть вычислена как число $\frac{1}{2 k}$-целых точек (т.е. точек с рациональными координатами, знаменатели которых равны $2 k$ ) полиэдра $\Delta_{g \Theta}$ по формуле Дюстермана-Экмана [8, гл. 3]; это число есть число Верлинде и равно размерности пространства конформных блоков уровня $k$ и рода $g$;

3) само же множество точек $\left(\Delta_{g \Theta}\right)_{2 k}=\frac{1}{2 k} \mathbb{Z}^{3 g-3} \cap \Delta_{g \Theta}=B S_{k}$ совпадает с множеством бор-зоммерфельдовых слоев расслоения $c_{\Delta_{g} \Theta}(2.2)$ уровня $k$.

В рамках симплектической геометрии мы имеем известную ситуацию: два расслоения с лагранжевыми слоями над одной и той же базой:

$$
\operatorname{ClRep}\left(\pi_{1}(\Sigma)\right) \stackrel{{ }^{c} \Delta_{g \Theta}}{\longrightarrow} \Delta_{g \Theta} \stackrel{m}{\longleftarrow} D M_{g}
$$

где $m$ - отображение моментов для регулярного действия тора многообразия Дельцана.

Такую конфигурацию уместно сравнить с "зеркальными расслоениями”.

Напомним, что гипотетически SYZ-зеркальная конструкция [11] также состоит из двух (дуальных) лагранжевых расслоений над одной и той же базой. Как и в нашем случае, можно рассматривать оба расслоения как семейства лагранжевых циклов с вырождениями.

Левое семейство

$$
\operatorname{ClRep}\left(\pi_{1}(\Sigma)\right) \stackrel{c_{\Delta_{g} \Theta}}{\rightarrow} \Delta_{g \Theta}
$$

имеет слои одинаковой размерности с особыми слоями над $(3 g-4)$-остовом базы.

Правое семейство

$$
D M_{g} \stackrel{m}{\rightarrow} \Delta_{g \Theta}
$$

имеет слоями торы разной размерности. А именно, ecли $\operatorname{sk}_{i}\left(\Delta_{g \Theta}\right)-i$-мерный остов в $\partial \Delta_{g \Theta}$, то

$$
p \in \operatorname{sk}_{i}\left(\Delta_{g \Theta}\right) \backslash \operatorname{sk}_{i+1}\left(\Delta_{g \Theta}\right) \Longrightarrow m^{-1}(p)=T^{i}
$$

есть $i$-мерный тор. Более того, каждая $i$-мерная грань $F_{i}$ определяет проективное подпространство $\mathbb{P}^{i}\left(F_{i}\right) \subset D M_{g}$ с действием $i$-тора, которое само есть пространство Дельцана. Следовательно, в дельцановской модели $D M_{g}$ лежит конфигурация проективных подпространств, соответствующих уменьшению размерности слоя. Это - типичное поведение изотропных слоев предквантованной динамической системы. 


\section{§7. Конформные блоки}

Итак, для любой комплексной кривой $\Sigma$ мы имеем два компактных комплексных поляризованных многообразия $\left(M_{\Sigma}(2,0), \Theta\right)$ и $\left(D M_{g}, H\right)$ с пространствами сечений $H^{0}\left(M_{\Sigma}(2,0), \Theta^{k}\right)$ и $H^{0}\left(D M_{g}, H^{k}\right)$ одинаковой размерности.

Следующая конструкция устанавливает прямую связь между этими пространствами. Прежде всего отметим следуюший простой геометрический факт.

ПРЕДЛОЖЕНИЕ 7.1. Полиәдр $\Delta_{g \Theta}$ имеет один иентр $c_{0}$ иентральной симметрии.

Очевидно, что слои обоих расслоений (6.2) над этим центром регулярны.

Вблизи регулярных слоев $c_{g \Theta}^{-1}\left(c_{0}\right), m^{-1}\left(c_{0}\right)$ можно отождествить наши торические пространства с помошью эквивариантных координат Дарбу-Вайнстейна, в частности отождествить слои

$$
c_{g \Theta}^{-1}\left(c_{0}\right)=m^{-1}\left(c_{0}\right)=T^{3 g-3} .
$$

Торы обоих семейств лагранжевы, так что ограничения поляризаций $\Theta_{\mid c_{g \Theta}^{-1}\left(c_{0}\right)}$ и $H_{\mid m^{-1}\left(c_{0}\right)}$ являются тривиальными линейными расслоениями с плоскими связностями, которые калибровочно эквивалентны между собой. (Легко видеть, что эквивариантная лемма Дарбу-Вайнстейна может быть продолжена до идентификации линейных расслоений с унитарными связностями при отождествлении (7.1).)

Учитывая все предыдушие факты, получим тор $T_{0}^{3 g-3}$, снабженный тривиальным линейным расслоением с плоской связностью $\left(L_{0}, a_{0}\right)$ (суперцикл) и лагранжевы вложения

$$
\operatorname{ClRep}\left(\pi_{1}(\Sigma)\right) \supset c_{g \Theta}^{-1}\left(c_{0}\right) \hookleftarrow T_{0}^{3 g-3} \hookrightarrow m^{-1}\left(c_{0}\right) \subset D M_{g},
$$

так что прообразы поляризаций $\Theta$ и $H$ равны $(L, a)$.

Тогда отображения ограничений

$$
H^{0}\left(M_{\Sigma}(2,0), \Theta^{k}\right) \rightarrow \Gamma^{\infty}\left(L_{0}\right) \leftarrow H^{0}\left(D M_{g}, H^{k}\right)
$$

являются вложениями и дают идентификацию пространств голоморфных сечений.

Следовательно, окрестности неособых точек пространства $\operatorname{ClRep}\left(\pi_{1}(\Sigma)\right)$ с гладким действием тора моделируются линейным действием тора на комплексном проективном пространстве, как и предписывает эквивариантная лемма Дарбу- Вайнстейна. Для особых точек необходимо найти новые локальные модели, обобщающие случай $\mathbb{C P}^{n}$.

На связь неабелевых тэта-функций с теоремой Дельцана автору указал Ж. Мурао в процессе совместной работы (вместе с К. Флорентино и Ж. Нуньесом) над конструкцией неабелевых тэта-функций при помощи обобщенной конструкции Сигала CST. Автор выражает всем им глубокую благодарность, а также благодарит У. Оксбери и особенно М. Рида за уточнение условия (1.1) и Институт высших исследований Кореи (KIAS, Seoul) за поддержку и гостеприимство во время написания этой работы. 


\section{Список литературы}

1. Delzant T. Hamiltoniens périodiques et images convexes de l'application moment // Bull. Soc. Math. France. 1988. V. 116. P. 315-339.

2. Goldman $W$. The symplectic nature of fundamental groups of surfaces // Adv. in Math. 1984. V. 54. P. 200-225.

3. Donaldson S. Gluing techniques in the cohomology of moduli spaces // Topological methods in modern mathematics (Stony Brook, 1991). Houston: Publish or Perish, 1993. P. 137-170.

4. Tyurin A.N. Three mathematical facets of SU(2)-spin networks. Preprint 35/2000 of Instituto Superior Tecnico, Lisbon, math.DG/0011035.

5. Fulton W. Introduction to toric varieties // Ann. of Math. Studies. V. 131. Princeton: Princeton University Press, 1993.

6. Tyurin A. N. Lattice gauge theory and the Florentino conjecture. Preprint 5/2001 of Instituto Superior Tecnico, Lisbon, math.DG/0102118.

7. Jeffrey L. C., Weitsman J. Bohr-Sommerfeld orbits in the moduli space of flat connections and the Verlinde dimension formula // Commun. Math. Phys. 1992. V. 150. P. 593-630.

8. Guillemin $V$. Moment maps and combinatorial invariants of Hamiltonian $T^{n}$-spaces. Boston-Basel-Berlin: Birkhäuser, 1994.

9. Audin M. Lectures on gauge theory and integrable systems // Gauge theory and symplectic geometry (Montreal, 1995). Kluwer: NATO ASI Series, 1997. P. 1-48.

10. Florentino $C$. Symmetries and moment polyhedrons. Preprint. Stony-Brook, 1999.

11. Strominger A., Yau S.-T., Zaslow E. Mirror symmetry is T-duality // Nucl. Phys. 1996. V. 479. P. 243-259.

12. Jeffrey L. C., Hurtubise J. C. Representations with Weighted Frames and Framed Parabolic Bundles // Canad. J. Math. 2000. V. 52. № 6. P. 1235-1268.

Поступило в редакцию

10.IX.2001 